Correspondence: P.E. Van Schil, Dept of Thoracic and Vascular Surgery, Antwerp University Hospital, Wilrijkstraat 10, B-2650 Edegem, Antwerp, Belgium. E-mail: paul.van.schil@uza.be

Statement of Interest: None declared.

\section{REFERENCES}

1 Lara JF, Catroppo JF, Kim DU, et al. Dendriform pulmonary ossification, a form of diffuse pulmonary ossification: report of a 26year autopsy experience. Arch Pathol Lab Med 2005; 129: 348-353.

2 Jaderborg JM, Dunton RF. Rare clinical diagnosis of dendriform pulmonary ossification. Ann Thorac Surg 2001; 71: 2009-2011.

3 Felson B, Schwarz J, Lukin RR, et al. Idiopathic pulmonary ossification. Radiology 1984; 153: 303-310.

4 Capelli A, Lusuardi M, Cerutti CG, et al. Lung alkaline phosphatase as a marker of fibrosis in chronic interstitial disorders. Am J Respir Crit Care Med 1997; 155: 249-253.
5 Chan ED, Morales DV, Welsh CH, et al. Calcium deposition with or without bone formation in the lung. Am J Respir Crit Care Med 2002; 165: 1654-1669.

6 Joines RW, Roggli VL. Dendriform pulmonary ossification. Report of two cases with unique findings. Am J Clin Pathol 1989; 91: 398-402.

7 Colby TV. Miscellaneous conditions and lung diseases of unknown origin. In: Hasleton PS, ed. Spencer's Pathology of the Lung. New York, McGraw-Hill, 2009; pp. 792-795.

8 Abedin M, Tintut Y, Demer LL. Vascular calcification: mechanisms and clinical ramifications. Arterioscler Thromb Vasc Biol 2004; 24: 1161-1170.

9 Erasmus JJ, Connolly JE, McAdams HP, et al. Solitary pulmonary nodules: part I. Morphologic evaluation for differentiation of benign and malignant lesions. Radiographics 2000; 20: 43-58.

10 Leef JL III, Klein JS. The solitary pulmonary nodule. Radiol Clin North Am 2002; 40: 123-143.

\title{
An autopsy case of pulmonary veno-occlusive disease refractory to imatinib
}

\section{To the Editors:}

Pulmonary veno-occlusive disease (PVOD) is a rare variant of pulmonary hypertension (PH). PVOD is reportedly refractory to the majority of $\mathrm{PH}$-specific vasodilators; however, a recent case report demonstrated that a tyrosine kinase inhibitor, imatinib, markedly improved functional capacity in a patient with PVOD [1]. The present case report documents clinical and haemodynamic effects of imatinib in a patient with pathologically confirmed PVOD.

In 2007, a 73-yr-old female experienced progressive exertional dyspnoea and was diagnosed with $\mathrm{PH}$ in a community hospital. Diuretics and beraprost (oral prostanoid) were administered to no avail. The patient was referred to Hokkaido University Hospital (Sapporo, Japan) in November 2008. She had never smoked and had no occupational or environmental inhalation history.

Functional capacity was New York Heart Association class III. Blood pressure was 110/65 mmHg, and coarse crackles were present in both lungs. Serum aspartate aminotransferase and alanine aminotransferase levels were within normal range, whereas hepatitis B (HB) surface and HBe antigens were both positive. Serum brain-type natriuretic polypeptide was elevated at 2,456 $\mathrm{pg} \cdot \mathrm{mL}^{-1}$, and arterial blood gas analysis showed hypoxaemia (arterial oxygen tension $55.2 \mathrm{mmHg}$ ) and hypocapnia (arterial carbon dioxide tension $33.9 \mathrm{mmHg}$ ). Chest radiography demonstrated marked cardiomegaly, dilated main pulmonary artery and pulmonary congestion. Echocardiography showed marked right atrial and ventricular enlargement and severe tricuspid regurgitation. Pulmonary function tests revealed normal forced vital capacity (FVC), forced expiratory volume in $1 \mathrm{~s}$ (FEV1) and FEV1/FVC, whereas diffusing capacity of the lung for carbon monoxide
(DL,CO) and DL,CO/alveolar volume (VA) were markedly reduced: $D \mathrm{~L}, \mathrm{CO} 4.16 \mathrm{~mL} \cdot \mathrm{min}^{-1} \cdot \mathrm{mmHg}^{-1}, 33.5 \%$ of predicted; $D \mathrm{~L}, \mathrm{CO} / V \mathrm{~A} 1.61 \mathrm{~mL} \cdot \mathrm{min}^{-1} \cdot \mathrm{mmHg}^{-1} \cdot \mathrm{L}^{-1}, 37.4 \%$ pred. High-resolution computed tomography (HRCT) revealed slight but diffuse ground-glass opacities, thickening of the septal lines, and hilar and mediastinal lymphadenopathy (fig. 1). Right heart catheterisation performed at this time exhibited elevated mean pulmonary artery pressure $\left(\bar{P}_{\mathrm{pa}}\right)$ and pulmonary vascular resistance (PVR) along with slightly reduced cardiac output (CO): $\bar{P}_{\text {pa }} 53 \mathrm{mmHg}$, pulmonary capillary wedge pressure $10 \mathrm{mmHg}, \mathrm{CO} 3.45 \mathrm{~L} \cdot \mathrm{min}^{-1}$ (cardiac index $2.63 \mathrm{~L}$. $\mathrm{min}^{-1} \cdot \mathrm{m}^{-2}$ ), right ventricular end diastolic pressure $14 \mathrm{mmHg}$, right atrial pressure $7 \mathrm{mmHg}$, and PVR 1,159 dyn $\cdot \mathrm{s} \cdot \mathrm{cm}^{-5}$.

With a clinical diagnosis of PVOD, an oral phosphodiesterase-5 inhibitor, sildenafil, was started in December 2008 but hypoxia and pulmonary haemodynamics deteriorated. The endothelin receptor antagonist bosentan was not initiated because of comorbid HBe antigen-positive HB. The intravenous prostacyclin epoprostenol was not used because we feared a lifethreatening deterioration of pulmonary oedema and hypoxaemia, although a recent report suggested that it can have a beneficial effect on PVOD when used with caution [2]. Lung transplantation is the optimal treatment for PVOD, and we considered the patient's suitability for this procedure; however, we concluded that the patient would not be a candidate because of her age, poor functional status, estimated long waiting time and the possibility of relapse of multiple myeloma. After reading a recent case report in which a patient with typical features of PVOD responded well to imatinib [1], we decided to use this agent in our patient. We administered imatinib (Gleevec ${ }^{\circledR}$; Novartis Pharmaceuticals, Basle, Switzerland) in February 2009 after obtaining approval from the ethical committee of our institution and written informed 

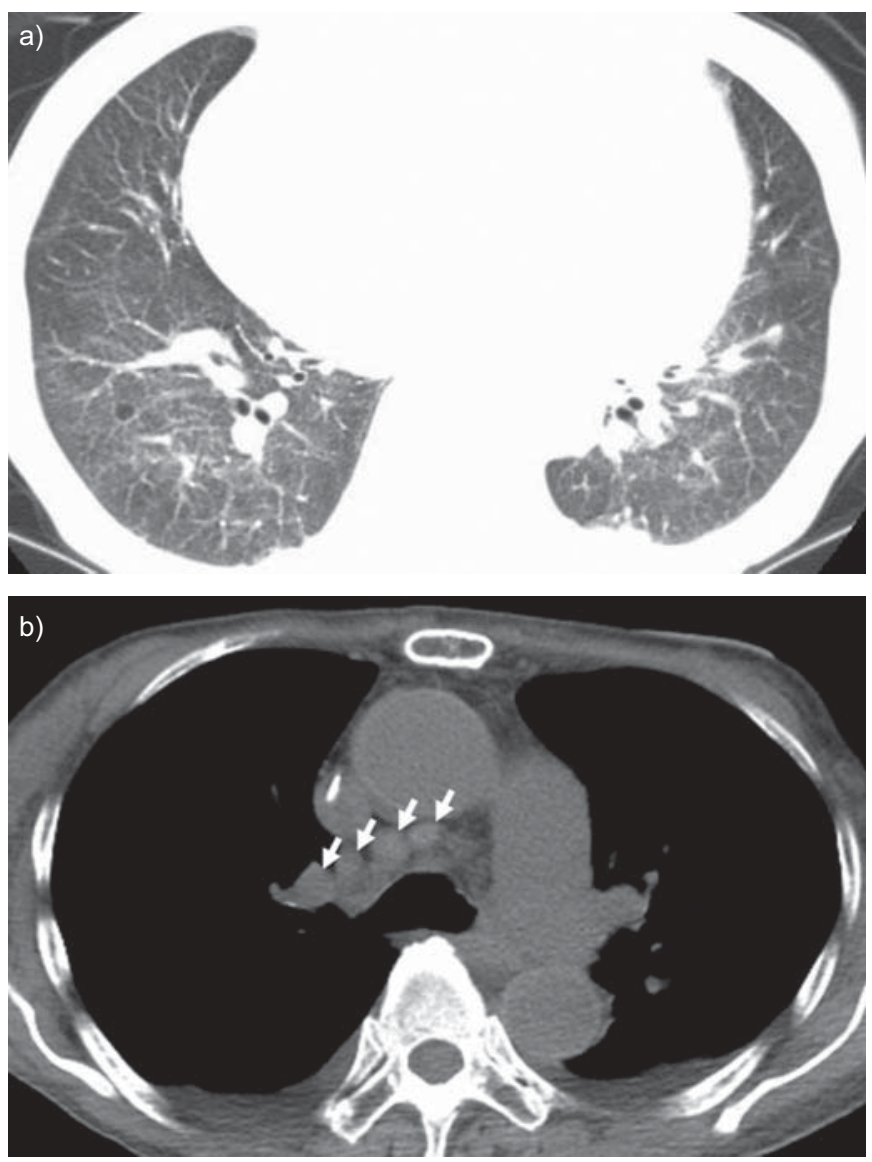

FIGURE 1. a) High-resolution computed tomography scan of the chest showing diffuse ground-glass opacities and thickened interlobular septa. b) Hilar and mediastinal lymphadenopathy is also noted (arrows).

consent from the patient, with the aim of improving pulmonary haemodynamics without worsening pulmonary congestion and oxygenation. The starting dose was $50 \mathrm{mg}$ daily, increasing to $100 \mathrm{mg}$ daily after 2 weeks. During the following 3 months, however, the patient's functional capacity gradually deteriorated. Moreover, 12 weeks after the start of imatinib right heart catheterisation showed slight worsening of measurements ( $\bar{P}_{\text {pa }} 50 \mathrm{mmHg}, \mathrm{CO} 2.74 \mathrm{~L} \cdot \mathrm{min}^{-1}$, PVR 1,343 dyn $\left.\cdot \mathrm{s} \cdot \mathrm{cm}^{-5}\right)$ as compared with those before treatment $\left(\bar{P}_{\mathrm{pa}}\right.$ $49 \mathrm{mmHg}$, CO $3.17 \mathrm{~L} \cdot \mathrm{min}^{-1}$, PVR 1,136 dyn $\cdot \mathrm{s}^{\cdot} \mathrm{cm}^{-5}$ ). Imatinib was reduced to $50 \mathrm{mg}$ daily because of insufficient efficacy and pancytopenia. The patient deteriorated further and died of right heart failure in June 2009.

At autopsy, gross findings were marked dilatation of the right atrium and ventricle, wall thickening of the right ventricle, and compression of the left ventricle by a displaced interventricular septum. There was also a small amount of pericardial effusion. Microscopically, there was fibrotic obstruction of the veins/ venules (fig. 2a) and advanced arterial narrowing with medial hypertrophy and intimal thickening, both of which were broadly distributed throughout the lungs. Haemangiomatous change of the pulmonary capillaries was also noted (fig. $2 b$ ). Immunohistochemical studies for platelet-derived growth factor-receptor (PDGF-R)- $\beta$ was negative in diseased venous / venular walls, although there was positive staining in the spindle-shaped pericytes at the perivascular location (fig. 2c). The staining was negative in arterial endothelial cells and in bronchiolar epithelial cells (fig. 2d). However, there was weak but positive staining in the hypertrophied arterial media and in the bronchiolar smooth muscle cells (fig. 2d). PDGF-BB (a ligand of PDGF-R- $\beta$ ) expression was seen in type II pneumocytes (fig. 2e) and in macrophages, although it was not visible in venous/venular, arterial and bronchiolar walls.

The present report describes histologically confirmed PVOD that did not respond either to pulmonary arterial hypertension (PAH)

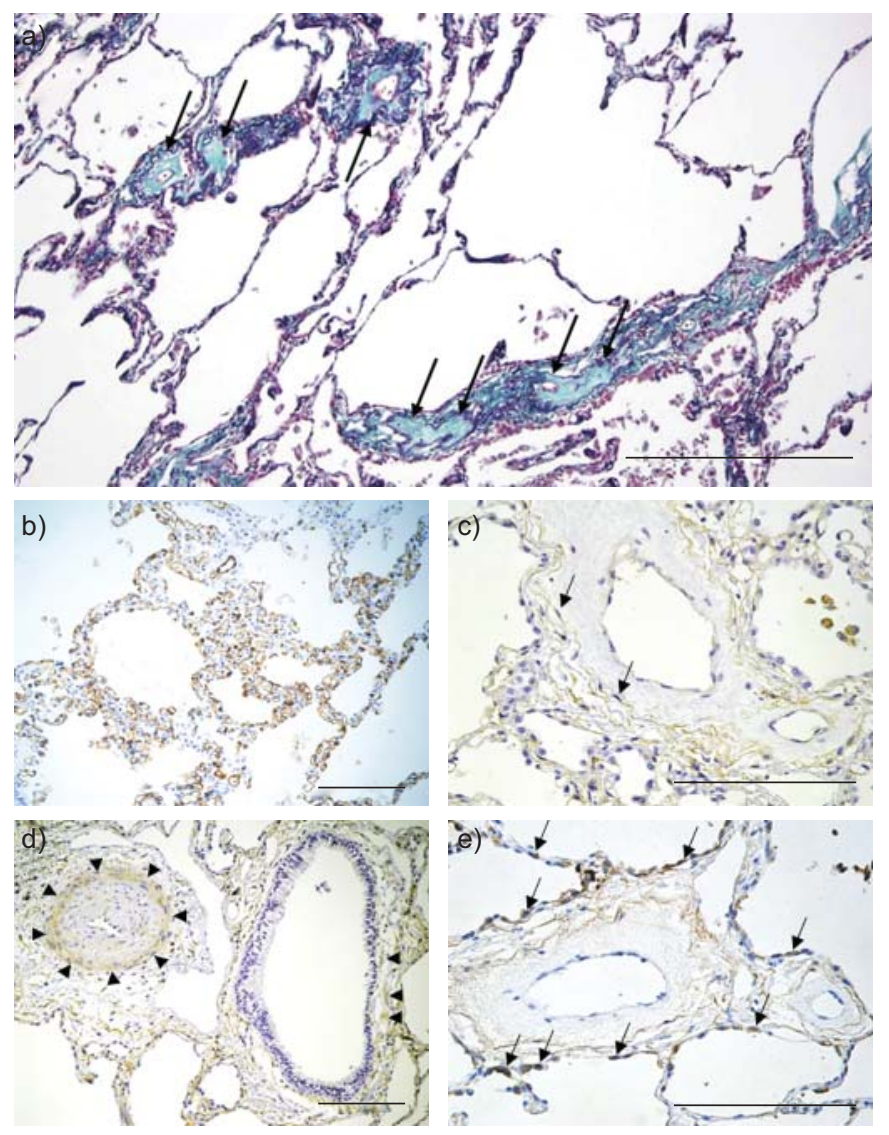

FIGURE 2. a) Fibrous obstruction and stenosis of pre-septal venules and veins (arrows; Elastica-Masson trichrome staining). Scale bar $=500 \mu \mathrm{m}$. b) CD31 immunohistochemistry reveals disordered capillary growth, or haemangiomatous change, in alveolar walls. Rabbit polyclonal anti-CD31 antibody dilution 1:50

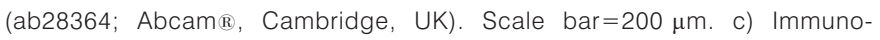
histochemistry for platelet-derived growth factor-receptor (PDGF-R)- $\beta$ is negative in diseased venous/venular walls, whereas it showed positive staining for pericytes at the perivascular location (arrows; monoclonal rabbit anti-PDGF-R- $\beta$ (C82A3) antibody dilution 1:200; Cell Signaling Technology, Danvers, MA, USA). Scale bar $=200 \mu \mathrm{m}$. d) Immunohistochemistry for PDGF-R- $\beta$ is negative in arterial endothelial cells and also in bronchiolar epithelial cells. However, there was weak but positive staining in the arterial smooth muscle cells (particularly in the outer fifth to quarter of the media) and in the bronchiolar smooth muscle cells (arrowheads; monoclonal rabbit anti-PDGF-R- $\beta$ antibody dilution 1:200 (C82A3); Cell Signaling Technology). Scale bar $=200 \mu \mathrm{m}$. e) Immunohistochemistry for PDGF-BB. No staining was observed in the remodelled vessels, although type II pneumocytes (arrows) and macrophages revealed positive staining (arrows; polyclonal rabbit anti-PDGF-BB antibody dilution 1:10 (ab9704); Abcam). Scale bar $=200 \mu \mathrm{m}$. 
specific therapies or to imatinib. Post mortem immunohistochemical studies showed the absence of PDGF-R- $\beta$ expression in pulmonary veins and venules.

Previous studies have shown that PVOD can be clinically distinguished from other types of $\mathrm{PH}$. Features suggestive of PVOD include markedly reduced DL,CO, and chest HRCT findings such as ground-glass opacities and thickening of septal lines [3, 4]. Poor or even adverse responses to PAHspecific vasodilators also suggest PVOD $[3,5]$. The present case correspondeds well to these clinical features.

Pathologically, PVOD is characterised by fibrotic obstruction of pulmonary veins/venules and by pulmonary capillary congestion with the presence of haemosiderin-laden macrophages $[4,6]$, which were identified in the present case. Interestingly, LANTUEJOUL et al. [6] reported that pathological characteristics of PVOD and pulmonary capillary haemangioma $(\mathrm{PCH})$ frequently coexist in lungs clinically or pathologically diagnosed with PVOD or PCH. Indeed, changes suggestive of PCH were identified in the present case. Narrowed pulmonary arteries with intimal and medial thickening were also noted in the present case, and may be secondary to venous/capillary obstruction or represent broad pulmonary vasculopathy distributed from artery through vein [7].

Imatinib is a molecular target drug that inhibits tyrosine kinase, an enzyme with critical functions in signalling involving molecules such as PDGF, bcr-abl and c-kit. It is widely used for the treatment of chronic myeloid leukaemia [8]; however, a recent report demonstrated that it markedly improved functional status and chest computed tomography findings in a patient with PVOD [1]. Its efficacy has also been noted in patients with advanced PAH $[9,10]$, although this is not necessarily observed in all patients with $\mathrm{PAH}$ [11]. In the present case, no favourable effect of imatinib was noted, at least, in part, because of absent PDGF-R expression in the diseased vessels. In fact, the presence of PDGF- $\beta$ receptors in remodelled vasculature has been reported in $\mathrm{PAH}$ [12]; however, it is unknown whether PDGF contributes to the development and progression of PVOD. Another possibility is that imatinib was not effective because the vessel disease had progressed to the stage of fibrosing/hyalinising obstruction.

In conclusion, the present case report indicates that imatinib does not necessarily provide a favourable outcome in all patients with PVOD. Better understanding and further evaluations of PVOD are needed to improve treatment strategies.

\section{H. Koiwa*, I. Tsujino*, D. Ikeda*, H. Ohira*, M. Tanino" and M. Nishimura}

*First Dept of Medicine, Hokkaido University Hospital, "Laboratory of Cancer Research, Dept of Pathology, Hokkaido University Graduate School of Medicine, and "Division of Respiratory Medicine, Dept of Internal Medicine, Hokkaido University Graduate School of Medicine, Sapporo, Japan.

Correspondence: I. Tsujino, First Dept of Medicine, Hokkaido University Hospital, North 14, West 5, Kita-ku, Sapporo, Sapporo 060-8648, Japan. E-mail: itsujino@med.hokudai.ac.jp

Statement of Interest: None declared.

\section{REFERENCES}

1 Overbeek MJ, van Nieuw Amerongen GP, Boonstra A, et al. Possible role of imatinib in clinical pulmonary veno-occlusive disease. Eur Respir J 2008; 32: 232-235.

2 Montani D, Jaïs X, Price LC, et al. Cautious epoprostenol therapy is a safe bridge to lung transplantation in pulmonary veno-occlusive disease. Eur Respir J 2009; 34: 1348-1356.

3 Montani D, Achouh L, Dorfmuller P, et al. Pulmonary venoocclusive disease: clinical, functional, radiologic, and hemodynamic characteristics and outcome of 24 cases confirmed by histology. Medicine (Baltimore) 2008; 87: 220-233.

4 Montani D, Price LC, Dorfmuller P, et al. Pulmonary venoocclusive disease. Eur Respir J 2009; 33: 189-200.

5 Palmer SM, Robinson LJ, Wang A, et al. Massive pulmonary edema and death after prostacyclin infusion in a patient with pulmonary veno-occlusive disease. Chest 1998; 113: 237-240.

6 Lantuejoul S, Sheppard MN, Corrin B, et al. Pulmonary venoocclusive disease and pulmonary capillary hemangiomatosis: a clinicopathologic study of 35 cases. Am J Surg Pathol 2006; 30: 850857.

7 Pietra GG, Capron F, Stewart S, et al. Pathologic assessment of vasculopathies in pulmonary hypertension. J Am Coll Cardiol 2004; 43: Suppl. 12, 25S-32S.

8 Baccarani M, Cortes J, Pane F, et al. Chronic myeloid leukemia: an update of concepts and management recommendations of European LeukemiaNet. J Clin Oncol 2009; 27: 6041-6051.

9 Ghofrani HA, Seeger W, Grimminger F. Imatinib for the treatment of pulmonary arterial hypertension. N Engl J Med 2005; 353: 14121413.

10 Souza R, Sitbon O, Parent F, et al. Long term imatinib treatment in pulmonary arterial hypertension. Thorax 2006; 61: 736.

11 Ghofrani HA, Morrell NW, Hoeper MM, et al. Imatinib in pulmonary arterial hypertension patients with inadequate response to established therapy. Am J Respir Crit Care Med 2010; 182: $1171-1177$.

12 Perros F, Montani D, Dorfmuller P, et al. Platelet-derived growth factor expression and function in idiopathic pulmonary arterial hypertension. Am J Respir Crit Care Med 2008; 178: 81-88.

DOI: $10.1183 / 09031936.00095710$ 